\title{
Routing Field Service Officers with SIMULINK
}

\author{
$\underline{\text { Y.V.Ng }}{ }^{\text {and I.R.Monks }}{ }^{a}$ \\ ${ }^{a}$ City West Water, Footscray, Victoria, Australia \\ Email: YNg@citywestwater.com.au
}

\begin{abstract}
City West Water (CWW) operates water and sewerage networks and associated infrastructure in the central business district and inner and western suburbs of Melbourne, Australia. At times these assets fail requiring an immediate response ('responsive maintenance') prioritised by assessed urgency. As part of this response, an initial assessment is made by a Field Service Officer (FSO). The FSO determines the resources required to repair the failure and informs the Dispatcher who allocates a field crew to perform the repair. The company's responsive maintenance performance is recorded for each job and reported in CWW's Key Performance Indicator (KPI) reports for the CWW Board of Directors and the Essential Services Commission.
\end{abstract}

CWW has set itself the goal of keeping its process at or below current levels while continuing to provide a consistent level of service. This is driving CWW to look for cost efficiencies. Two such efficiencies that CWW has considered are risk based job priority zoning and more efficient FSO job assignment and routing. In order to test the impact of these initiatives a simulation model was constructed using MathWorks' SIMULINK ${ }^{\mathrm{TM}}$ software. The model uses data from CWW internal systems including priority, time and location for each job, as well as the assigned FSO. Travel times between jobs are approximated using centroids of Melways map grids. The model processes the job queue and makes FSO assignment decisions for the Dispatcher, allowing the effects of various job priority zoning rules to be measured and compared.

The first version of the model simulated the current FSO job assignment and rules showed similar or slightly better average response times when compared to historical data. The model was modified further to test the Risk Zone prioritisation of jobs with different response time rules. The results showed blending job urgency and risk prioritisation achieved desirable response times. This paper describes the approach used to develop risk based job priority zones and how SIMULINK ${ }^{\mathrm{TM}}$ block diagrams were used to build a simulation of the current routing process and test some alternative approaches to job prioritisation.

Keywords: $\quad$ SIMULINK ${ }^{\mathrm{TM}}$, MATLAB ${ }^{\mathrm{TM}}$, routing, simulation 


\section{INTRODUCTION}

City West Water (CWW) is a water retailer providing potable water, recycled water and waste water services to residential, commercial and industrial customers in Melbourne, Victoria and the city's inner and western suburbs. The supply network of approximately $4,600 \mathrm{~km}$ of water mains and 4,000 km of sewer mains vary in diameter, material and age - some were installed over 100 years ago. CWW has a responsive maintenance program to address failures on the network. Faults require timely responses to restore services and avoid additional costs resulting from delayed repairs.

CWW has set itself the goal of keeping its process at or below current levels while continuing to provide a consistent level of service. This is driving CWW to look for cost efficiencies. This study involved consideration of a risk based approach to job prioritisation and finding efficiencies in assigning and routing Field Service Officers (FSOs). FSOs provide a first response to failures on the network, where they assess the issue and identify the work required for rectification. Calls from the public reporting faults are taken by Operations staff. They record each fault's details and nominate its work priority based on the assessed urgency. Routing FSOs to jobs is based on the nominated priority and the fault reported time (first-in/first-out) basis.

A change to a risk based job prioritisation and to more complex but more efficient routing rules would mean a significant change to years of entrenched practices.

Use of priority as the first criteria for order of servicing jobs and time that the fault was reported as the second criteria means that FSOs move between jobs inefficiently, often passing other jobs that could be done along the way to the "next" job. Target response times are set for each priority level and the achieved times must be reported by CWW. In practice strict priority sequencing of jobs means that the targets are usually beaten and, year to year, this leads to the targets for the priority levels reducing and converging closer together. This is not desirable as it leads to ever increasing levels of service and commensurate cost increases, something which is counter to CWW"s overall objective of maintaining its level of service while reducing its costs.

The approach used for the study of risk zone prioritisation looked at the problem from three perspectives: data analysis, existing risk consequence mappings, and a survey of characteristics of each area (Melways ${ }^{\mathrm{TM}}$ street maps). The outcome of each of these studies was used to propose new priority zones. In order to measure the effect of new rules on response times a simulation model was written using SIMULINK ${ }^{\mathrm{TM}}$.

\section{RESPONSIVE MAINTENANCE JOBS}

Customers, plumbers and members of the public call the CWW Operations Control Centre (OCC) to report faults (Fig. 1) such as low pressure, water quality concerns, bursts and leaks, and issues with the sewerage system. The call taker creates a job in the work maintenance system, FOCUS, filling in location details and fault description. They assign a priority based on the urgency of the problem ( $\mathrm{P} 1=$ Urgent, $\mathrm{P} 2=$ less urgent, $\mathrm{P} 3=$ nonurgent) then send the details to the Dispatcher. The Dispatcher then assigns the job to one of the FSOs on duty.

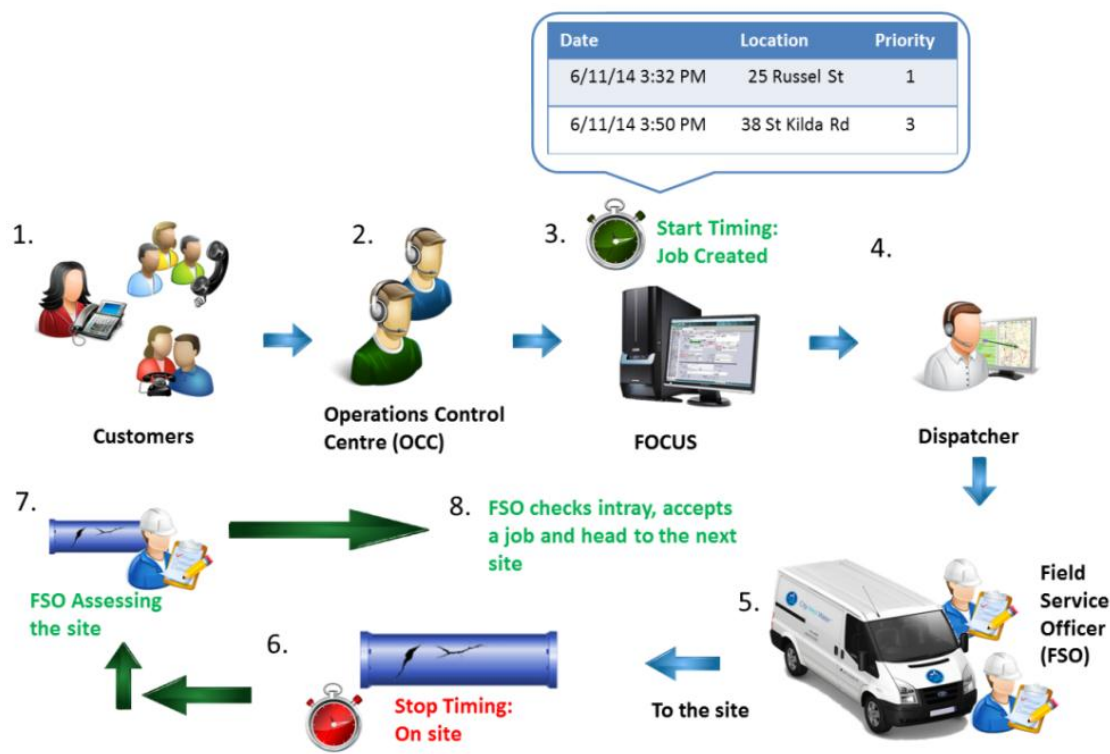

Figure 1: Fault logging and First Response Schematic 
The FSOs usually operate in loosely assigned territories and cover jobs of different priorities for both water and sewer. Within each territory there is normally sufficient work for the FSOs, they become familiar with the area and are within short travel distances should they be required at a job quickly. They move to jobs according to priority level and first-in/first-out within priority levels. The target response times for the different priority levels create time windows during which other work could be performed without necessarily breaching the target.

Table 1: Sample of the Input file for Faults

\begin{tabular}{|c|c|c|c|c|c|c|c|c|}
\hline Job\# & DateCreated & Priority & Location & OnSiteTime & SHORTCODE & FSO ID OriginalIntray & ONSITE_AVAIL_DATETIME & ENR_ONSITE_DATETIME \\
\hline 614774 & 27/04/2014 7:38 AM & 4 & 28 & 240 & SERV & attardb1 & 6/06/2014 1:34 PM & 3/06/2014 12:45 PM \\
\hline 617925 & 13/05/2014 10:50 AM & 4 & 42 & 5 & LEAK & attardb1 & 5/06/2014 2:01 PM & 5/06/2014 1:57 PM \\
\hline 620257 & 24/05/2014 5:52 PM & 4 & 28 & 5 & WOAF & durrana1 & 6/06/2014 8:03 AM & 6/06/2014 7:59 AM \\
\hline 621601 & 1/06/2014 8:04 AM & 3 & 42 & 33 & BURST & urbanig1 & 1/06/2014 10:42 AM & 1/06/2014 10:09 AM \\
\hline 621629 & 1/06/2014 6:05 PM & 2 & 28 & 21 & SISF & urbanig1 & 1/06/2014 6:51 PM & 1/06/2014 6:30 PM \\
\hline 621639 & 1/06/2014 11:55 PM & 1 & 56 & 25 & BLOCH & argeroj1 & 2/06/2014 12:59 AM & 2/06/2014 12:34 AM \\
\hline 621866 & 2/06/2014 9:46 AM & 1 & 42 & 17 & SERV & attardb1 & 2/06/2014 10:24 AM & 2/06/2014 10:07 AM \\
\hline 621937 & 2/06/2014 11:35 AM & 3 & 43 & 5 & SERV & izlemel1 & 2/06/2014 1:16 PM & 2/06/2014 1:16 PM \\
\hline 621966 & 2/06/2014 12:07 PM & 3 & 28 & 7 & WISF & durrana1 & 2/06/2014 2:52 PM & 2/06/2014 2:45 PM \\
\hline 621982 & 2/06/2014 12:54 PM & 2 & 43 & 5 & HYDCW & izlemel1 & 2/06/2014 1:25 PM & 2/06/2014 1:25 PM \\
\hline 622022 & 2/06/2014 2:10 PM & 3 & 42 & 15 & METER & urbanig1 & 2/06/2014 2:54 PM & 2/06/2014 2:39 PM \\
\hline 622083 & 2/06/2014 4:21 PM & 4 & 43 & 5 & WISF & izlemel1 & 3/06/2014 2:47 PM & 3/06/2014 2:47 PM \\
\hline 622096 & 3/06/2014 6:44 AM & 1 & 56 & 17 & BURST & attardb1 & $3 / 06 / 2014$ 7:42 AM & $3 / 06 / 20147: 25$ AM \\
\hline 622131 & 3/06/2014 10:07 AM & 3 & 43 & 35 & WOAF & izlemel1 & 3/06/2014 11:21 AM & 3/06/2014 10:46 AM \\
\hline 622154 & $3 / 06 / 2014$ 11:10 AM & 3 & 43 & 5 & METER & izlemel1 & 3/06/2014 3:21 PM & 3/06/2014 3:21 PM \\
\hline
\end{tabular}

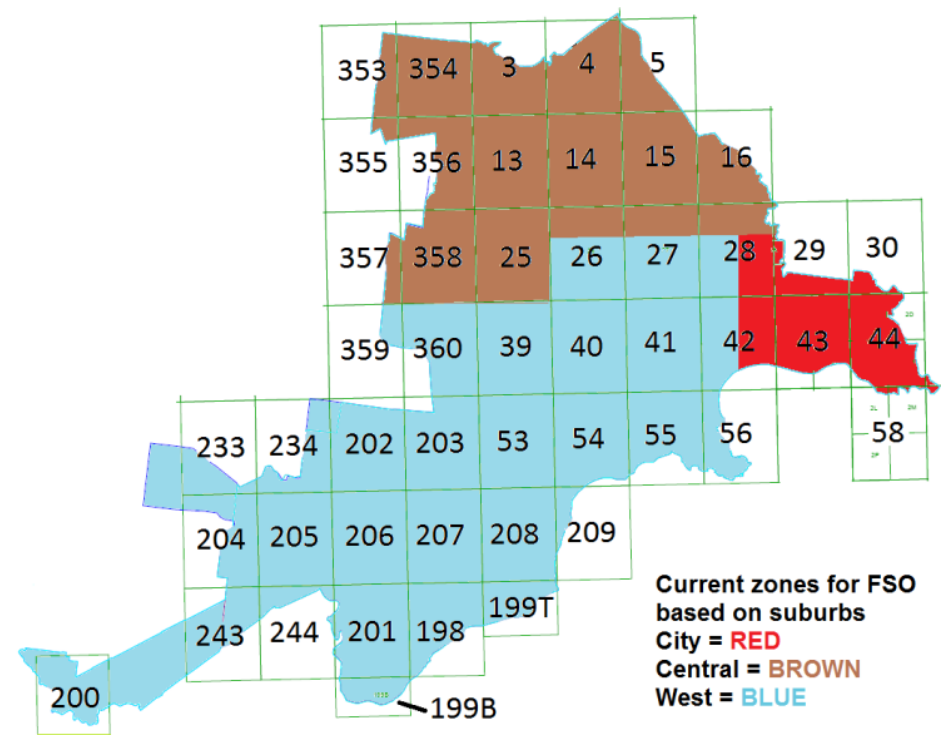

Figure 2: Current FSO Territories

\section{MODEL DEVELOPMENT}

\subsection{Identifying Job Priorities based on Risk Consequence Zones}

Jobs were analysed using three approaches to identify job priority zones based on risk.

Data was extracted from the Focus system for the analysis.

The location of each job was approximated to the centroid of its associated Melways' map grid, then plotted using MATLAB ${ }^{\mathrm{TM}}$ as a heatmap. Further analysis was done by splitting the job counts by map number, priority and hour of day. The heatmap (Fig. 3a) shows that Melbourne's central business district (CBD) area has the highest number of priority jobs followed by the immediate surrounding suburbs and then outer suburbs.

The second approach used existing risk consequence graphics for the assets of the sewer and water networks. The graphics colour code the network assets according to assessed risk consequence. Overlaying the Melways 
maps on these risk consequence graphics highlighted the risk level of the assets within each map and a comparison to the heatmap showed a high correlation.

The third approach was a survey of the characteristics of each of the Melways' map areas that scored twenty attributes for risk consequence. These three independent approaches lead to the identification of three zones: Melbourne CBD/inner city area was found to be the highest risk consequence zone, the older middle suburbs area forms the medium risk consequence zone and the outer most area comprising the newer suburbs and rural areas forms the lowest risk consequence zone (Fig. 3b). Jobs would receive priorities based on these three zones.

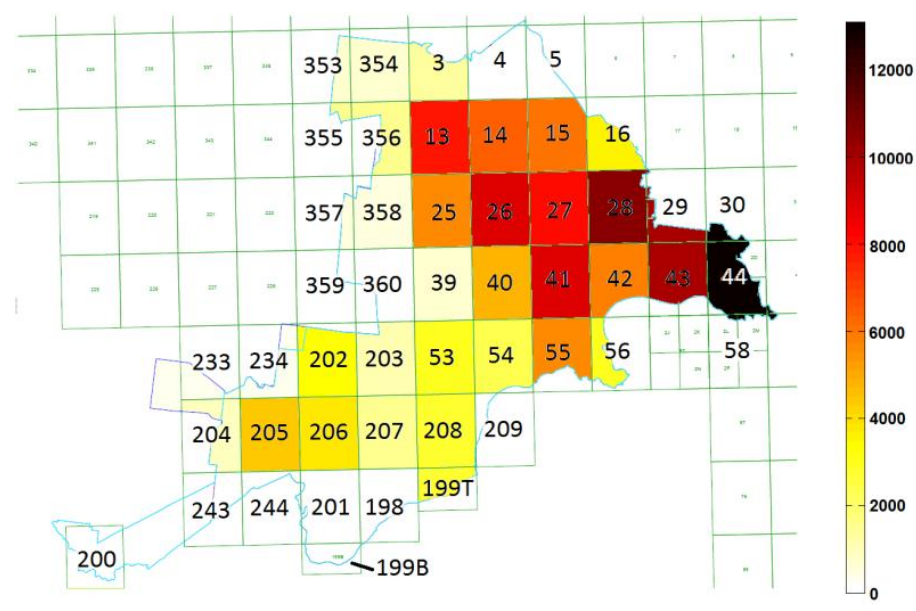

Figure 3a. Heatmap of the number of jobs reported in CWW licensed area by Melways map

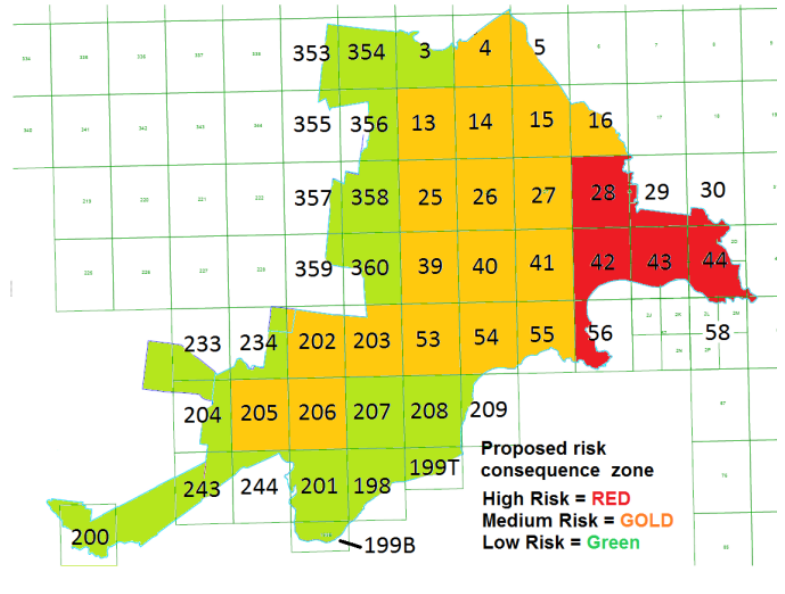

Figure 3b.The three risk consequence zones identified by the study

\subsection{Data}

The June 2014 period was selected as a representative sample of jobs and FSO shifts. The jobs data was extracted from the FOCUS system and used to test the simulation model. The job codes used in the simulation model were those for water network failures that are subject to "respond by" KPIs which measure the time between call taking and first arrival on site. Jobs are ordered by date and time created, attributes used in the model were: Date/time of jobs created, priority, FSO number, FSO roster, location, travel time between jobs and the time spent on the site assessing the failure and planning the rectification works before moving onto the next job (Table 1).

In the model, all FSOs on duty start their shift at the main depot in Melways' map 40 then split into three territories. The location of jobs was approximated using the relevant Melways map number. The travel time between Melways map grids was calculated by taking travel times between the centroids of map pairs. Consecutive jobs in the same map have a travel time set to map centroid to map boundary.

\subsection{Selection of SIMULINK for Modelling}

CWW selected SIMULINK ${ }^{\mathrm{TM}}$ and SimEvents ${ }^{\mathrm{TM}}$ for the project. SIMULINK ${ }^{\mathrm{TM}}$ provides functions to describe a problem visually and to simulate queue processes. MATLAB ${ }^{\mathrm{TM}}$ code can be integrated into the simulation for more complex functions. SimEvents ${ }^{\mathrm{TM}}$, the discrete-event simulation engine toolbox, was also used as it can model event-driven communication between components such as customised routing, processing delays, prioritization and other operations.

Using SIMULINK ${ }^{\mathrm{TM}}$ and SimEvents ${ }^{\mathrm{TM}}$, a series of block diagram models were built to simulate the FSO routing process. An iterative approach allowed the model to be built-up from a simple first model to include complex business logic.

\subsection{Early Stage Iterations}

In the first stage, the model was designed to prove the effectiveness of some basic SIMULINK ${ }^{\mathrm{TM}}$ modelling components. The model generated jobs, locations and priorities randomly, and then sorted according to the highest priority. All jobs were then assigned randomly to the FSOs on duty with the depot as a fixed starting location for travel to each job. The model established a basic simulation flow using an N-server block (ref. Table 2) to service the jobs by assigning FSOs. 
The second stage used two single server blocks (Table 2) representing two individual FSOs instead of the Nserver block. This allowed the modeller to view jobs that each FSO serviced (assessed). During the simulation process, the starting location of each FSO was set as the job location of the previous job so that the FSO travelled from job site to job site. The model also assigned jobs to FSOs based on the lower travelling time.

The third stage modified the model to more accurately reflect the current job assignment rules. It focused on one FSO and a list of jobs generated by MATLAB ${ }^{\mathrm{TM}}$ scripts, each with priority and location. The FSO's shift times were also implemented to allow the FSO to accept jobs when his shift started and put jobs on hold when his shift ended. In addition, timers were added into the model to count the time taken from when the job was created until the time that the FSO reached the site. The average job response time for each of the three priorities (P1, $\mathrm{P} 2$ and $\mathrm{P} 3$ ) was then calculated in MATLAB ${ }^{\mathrm{TM}}$.

Table 2: Descriptions of important SIMULINK ${ }^{\mathrm{TM}}$ blocks

\begin{tabular}{|l|l|l|l|}
\hline | & $\begin{array}{l}\text { Time-Based Entity Generator block: } \\
\text { Generate entities (Jobs) using } \\
\text { intergeneration times from a signal }\end{array}$ & $\begin{array}{l}\text { Event-Based sequence block: Generate event- } \\
\text { based sequence of numbers (Priority, } \\
\text { Location, Assess Time). }\end{array}$ \\
\hline
\end{tabular}

3.5 First working version

After the three early stage iterations, the first working version of the model was developed. It segmented the process into components to create a user friendly interface. The Data Store Memory block (Table 2) was used to store all the incoming jobs and the average job response time of each priority. The Data Store Read block (Table 2) then read the block and displayed the list.

The main platform of the model (Fig. 4) shows a list of jobs assigned to individual FSOs, the average response time for each priority and the final routing model where the job assigning process was implemented.

\subsection{Modified version for testing Risk Based Prioritisation scenarios}

The first working version of the model was modified to enable the Risk Based Prioritisation approach and alternate rules to be configured and tested. The scenarios covered prioritisation based on Job Urgency or Risk or a blend of the two and various Enroute (delay) time combinations. The scenarios and results are summarised in Table 3. In the scenarios only jobs with priorities 1, 2 and 3 were included as these are the jobs subject to required response time targets. Jobs with lower priorities would be used to fill any gaps between the higher priority jobs so were not required to be processed in this study.

\section{RESULTS}

The risk analysis has suggested a new approach to job prioritisation using zones to avoid additional costs from delayed response to network failures. The zoning can be used to influence a new routing strategy for FSOs. SIMULINK $^{\mathrm{TM}}$ and MATLAB ${ }^{\mathrm{TM}}$ proved appropriate tools for developing the simulation model using an iterative development methodology.

Response times generated through the first working simulation model of the current rules were compared with actual response times for the June 2014 data. The results showed that the average response times for each priority achieved using the current FSO territories were very close to, or had slightly better average response time compared to the real event. This indicated that the model accurately simulated current outcomes and would be useful for analysing new rules.

The model was then further modified to facilitate testing of the Risk Zone Priority approach (Table 3). 


\section{CONCLUSION}

The results from testing various scenarios and configuration rules showed that blending the job urgency priorities and risk based priorities could achieve graduated levels of service response times.

\section{ACKNOWLEDGEMENTS}

The authors acknowledge the contributions and advice of Daryl Ning, Principal Applications Engineer, The MathWorks, Sydney. The authors also acknowledge the contribution and support of staff from the Programmed Facility Management and City West Water.

Table 3: Results

FSO shifts:

\section{Weekdays}

Morning shift FSOs $=4$

Afternoon shift FSOs $=2$

Night shift FSOs $=1$

\section{$\underline{\text { FSO Routing Simulation Test Settings }}$}

Weekends

Morning shift FSOs $=2$

Night shift FSOs $=1$

Planned Enroute (Delay) Start Minutes - FSO is not required to commence travel to job until $m$ minutes after call

\begin{tabular}{|l|c|c|c|}
\hline Set 1 & Priority 1 & Priority 2 & Priority 3 \\
\hline All Risk Zones & 15 & 35 & 140 \\
\hline \multicolumn{4}{|l|}{ Priority 2 } \\
\hline Set 2 & Priority 1 & 30 & Priority 3 \\
\hline Ged Risk Zone 1 & 10 & 35 & 120 \\
\hline Green Risk Zone 3 & 15 & 45 & 140 \\
\hline
\end{tabular}

FSO Routing Target Times and Actual Response Times June 2014

\begin{tabular}{|l|c|c|c|}
\hline Average Response Times (minutes) & Priority 1 & Priority 2 & Priority $\mathbf{3}$ \\
\hline CWW Target Times & 24.5 & 45.0 & 313.8 \\
\hline Actual Response Times & 26.7 & 30.8 & 160.9 \\
\hline
\end{tabular}

FSO Routing Simulation Test Results

$\underline{\text { Scenario } 1}$ - Job Priority queue set to Job Urgency (ie. OCC assessed priority) + job type priority, with Planned Enroute Start time using Set 1 times.

job type : BURST, LEAK, HYDCL, HYDCW, VALVE were given higher priority within job urgency

\begin{tabular}{|l|c|c|c|}
\hline Average Response Times (minutes) & Priority 1 & Priority 2 & Priority 3 \\
\hline All Risk Zones & 22.6 & 39.4 & 147.4 \\
\hline
\end{tabular}

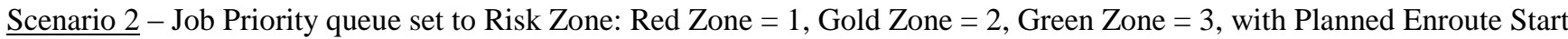
time using Set 1 times.

\begin{tabular}{|l|c|c|c|}
\hline Average Response Times (minutes) & Priority 1 & Priority 2 & Priority $\mathbf{3}$ \\
\hline All Risk Zones & 22.3 & 39.3 & 142.2 \\
\hline
\end{tabular}

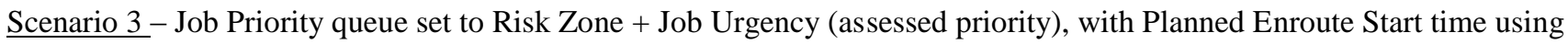

Set 1 times.

\begin{tabular}{|l|c|c|c|}
\hline Average Response Times (minutes) & Priority 1 & Priority 2 & Priority 3 \\
\hline Red Risk Zone 1 & 22.2 & 39.4 & 150.2 \\
\hline Gold Risk Zone 2 & 21.6 & 38.2 & 147.1 \\
\hline Green Risk Zone 3 & 22.5 & 38.5 & 140.5 \\
\hline
\end{tabular}

$\underline{\text { Scenario } 4}$ - Job Priority queue set to Job Urgency (Priority) + Risk Zone, with job type priority qualifier and with Planned Enroute Start time using Set 2 times.

job type : BURST, LEAK, HYDCL, HYDCW, VALVE were given higher priority within job urgency and Risk Zone

\begin{tabular}{|l|c|c|c|}
\hline Average Response Times (minutes) & Priority 1 & Priority 2 & Priority 3 \\
\hline Red Risk Zone 1 & 19.2 & 34.4 & 133.8 \\
\hline Gold Risk Zone 2 & 22.4 & 37.1 & 149.0 \\
\hline Green Risk Zone 3 & 24.0 & 48.4 & 197.8 \\
\hline
\end{tabular}




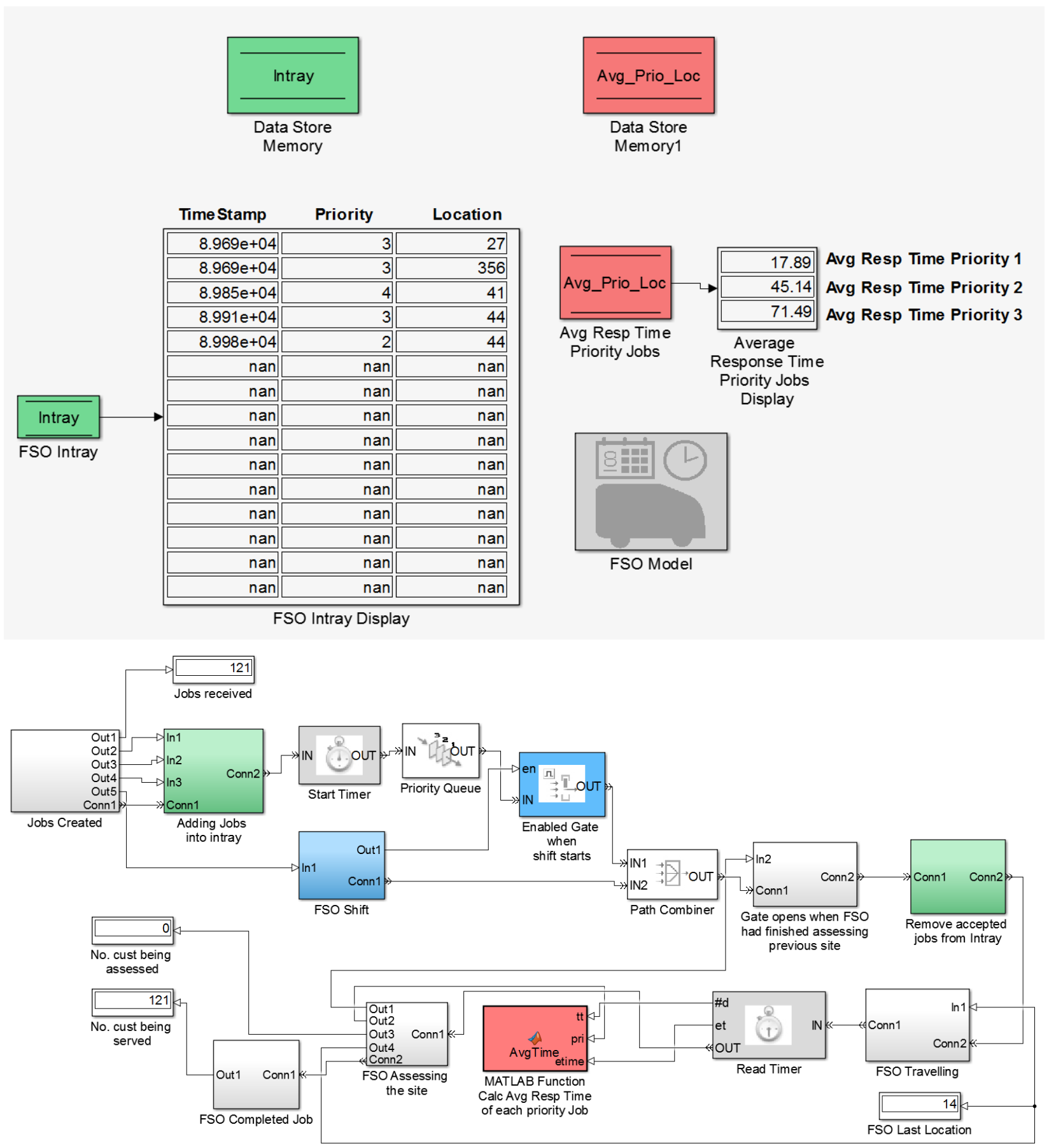

Figure 4: The first working version of the model 